\title{
METTL3 serves an oncogenic role in human ovarian cancer cells partially via the AKT signaling pathway
}

\author{
SHUMEI LIANG ${ }^{1}$, HONGWEI GUAN ${ }^{1}$, XIAOYAN LIN ${ }^{2}$, NA LI $^{1}$, FENG GENG $^{1}$ and JUAN LI ${ }^{1}$ \\ ${ }^{1}$ Department of Obstetrics and Gynecology, Shandong Provincial Hospital Affiliated to Shandong University, Jinan, \\ Shandong 250021; ${ }^{2}$ Department of Obstetrics and Gynecology, Weihai Huancui Maternal and \\ Child Care Family Planning Service Centre, Weihai, Shandong 264200, P.R. China
}

Received December 17, 2018; Accepted January 2, 2020

DOI: $10.3892 / \mathrm{ol} .2020 .11425$

\begin{abstract}
Methyltransferase-like 3 (METTL3) has been identified as a methyltransferase responsible for N6-methyladenosine (m6A) modification of mRNA. METTL3 functions in tumorigenesis and tumor development by promoting the translation of oncoproteins; however, the role of METTL3 in ovarian cancer has not been extensively studied. The present study performed immunohistochemistry to detect METTL3 expression levels in 52 samples of ovarian cancer tissue paired with corresponding paracancerous tissue. RNA interference was conducted to downregulate the expression levels of METTL3 in the SKOV3 and OVCAR3 ovarian cancer cell lines. Reverse transcription-quantitative PCR and western blot analysis demonstrated the effects of METTL3 knockdown on mRNA and protein levels, respectively. CCK- 8 , colony formation, apoptosis and Transwell assays were also performed. The results demonstrated that METTL3 exhibited significantly higher expression levels in ovarian cancer tissues compared with corresponding paracancerous tissue. High METTL3 expression levels were associated with large tumors, lymph node metastasis and high pathological grade. Cell proliferation analysis revealed that METTL3 knockdown reduced the proliferation and clonogenic ability of SKOV3 and OVCAR3 cells. Apoptotic rates were increased in METTL3-silenced ovarian cancer cells, which may have been mediated by the activation of the mitochondrial apoptosis pathway, and METTL3 knockdown reduced cell invasion. METTL3 knockdown downregulated the phosphorylation levels of AKT and the expression of the downstream effector Cyclin D1. These
\end{abstract}

Correspondence to: Dr Juan $\mathrm{Li}$, Department of Obstetrics and Gynecology, Shandong Provincial Hospital Affiliated to Shandong University, 324 Jingwuweiqi Road, Jinan, Shandong 250021, P.R. China E-mail:1_shumei1982@163.com

Abbreviations: METTL3, methyltransferase-like 3; m6A, N6-methyladenosine

Key words: METTL3, oncogene, apoptosis, mitochondria pathway, AKT results suggested that METTL3 may serve an oncogenic function in the progression of human ovarian cancer cells partially through the AKT signaling pathway, indicating that METTL3 may be a potentially novel therapeutic target for the treatment of ovarian cancer.

\section{Introduction}

Ovarian cancer is one of the most common gynecological tumors (1). Although surgical resection combined with platinum and taxane-based chemotherapy inhibits the development of tumors and improves short and medium-term survival, the 5 -year survival rate remains $<30 \%$ due to drug resistance and recrudescence worldwide (2). Therefore, exploring the underlying mechanisms of ovarian cancer development and metastasis may aid the identification of a novel therapeutic target.

N6-methyladenosine (m6A) is a highly conserved functional modification of RNA widely distributed in all eukaryotic cells (3). The m6A modification functions in a number of biological processes, such as mRNA post-transcriptional processing, location and translation; $\mathrm{m} 6 \mathrm{~A}$ is important in human diseases, including obesity and liver cancer development $(4,5)$. Catalyzation of the m6A modification is mediated by a methyltransferase complex consisting of three proteins: Methyltransferase-like 3 (METTL3), methyltransferase-like 14 and Wilms' tumor 1-associating protein (6). Functional studies have demonstrated that METTL3 is closely associated with the development of various tumors, and its functions (pro or anti-tumor) vary between different tumor types. For example, Lin et al (7) have reported that METTL3 functions as a translational promotor for multiple oncogenes, including epidermal growth factor receptor (EGFR) and tafazzin (TAZ), in lung cancer, contributing to tumor proliferation, survival and invasion. Reduced METTL3 expression levels in human myeloid leukemia cell lines induces cell differentiation and apoptosis, delaying leukemia progression in recipient mice in vivo (8). Li et al (9) have demonstrated that METTL3 functions as a tumor suppressor in the development of renal cell carcinoma by inhibiting proliferation and invasion. However, the function of METTL3 in human ovarian cancer remains unclear. 
The present study aimed to investigate METTL3 expression levels in different ovarian cancer tissue histotypes and analyze the functional effect of METTL3 knockdown in the human ovarian cancer cell lines SKOV3 and OVCAR3.

\section{Materials and methods}

Patient tissue samples. The present study was approved by The Ethics Committee in Shandong Provincial Hospital (Jinan, China) and all patients provided informed written consent. A total of 52 ovarian cancer tissue and adjacent normal tissue specimens were collected from patients diagnosed with ovarian cancer and treated at Shandong Provincial Hospital between February 2018 and March 2019. All patients were aged between 35 and 67 years and had only undergone surgery, receiving no other treatment. The clinicopathological data of patients obtained included age, tumor size, tumor site, lymph node metastasis and clinical stages (Table I).

Immunohistochemistry. The ovarian cancer tissues and adjacent paracancer tissues were stained using an EliVision ${ }^{\mathrm{TM}}$ Plus kit (Fuzhou Maixin Biotech Co., Ltd.) according to the manufacturer's protocol. Images were obtained using an upright light microscope system (Nikon Corporation; magnifications, $\mathrm{x} 100$ and $\mathrm{x} 400$ ). The METTL3 immunostaining score was the sum of the staining intensity score and the positive staining cell rate score. The staining intensity was scored as follows: no staining, 0 ; weak staining, 1; moderate staining, 2; and strong staining, 3 . The positive staining cell rate was scored as follows: 0 to $5 \%, 0 ; 5$ to $25 \%, 1 ; 26$ to $50 \%, 2 ; 51$ to $75 \%, 3$; and $>75 \%, 4$. A score $<2$ points was considered low METTL3 expression, whereas $>3$ points was considered high METTL3 expression.

Ovarian cancer cell culture and transfection. Human ovarian cancer cell lines SKOV3 and OVCAR3 were purchased from The Cell Bank of Type Culture Collection of the Chinese Academy of Sciences. The cells were cultured in Dulbecco's Modified Eagle's Medium (DMEM; HyClone; GE Healthcare Life Sciences) supplemented with 10\% FBS (Gibco; Thermo Fisher Scientific, Inc.) at $37^{\circ} \mathrm{C}$ in $5 \% \mathrm{CO}_{2}$. Short hairpin (sh) RNAs (pSUPER) shRNA1, shRNA2 and shRNA3 targeting METTL3 and a control shRNA were designed and synthesized by Shanghai GenePharma Co., Ltd. Ovarian cancer cells were seeded into a 6-well plate and cultured to logarithmic phase for $24 \mathrm{~h}$ at $37^{\circ} \mathrm{C}$. shRNA transfection was performed using Lipofectamine ${ }^{\circledR} 2000$ (Invitrogen; Thermo Fisher Scientific, Inc.). Following transfection for $48 \mathrm{~h}$, METTL3 mRNA expression levels were determined using reverse transcription-quantitative (RT-q) PCR.

$R T-q P C R$. Total RNA of human ovarian cancer cells was prepared using an Ultrapure RNA Extraction kit (CWBio) according to the manufacturer's protocol. A total of $1 \mu \mathrm{g}$ RNA was reverse-transcribed into cDNA with random primers using a HiFiScript cDNA Synthesis kit (CWBio) according to the manufacturer's protocol. The reverse transcription reaction conditions were as follows: incubation at $42^{\circ} \mathrm{C}$ for $50 \mathrm{~min}$, followed by incubation at $85^{\circ} \mathrm{C}$ for $5 \mathrm{~min}$ to terminate the reaction. METTL3 mRNA expression levels were determined by fluorescence qPCR using UltraSYBR mixture (CoWin Biosciences) according to the manufacturer's protocol and an Applied Biosystems 7500 FAST Real-time PCR system (Applied Biosystems; Thermo Fisher Scientific, Inc.). The following thermocycling conditions were used for qPCR: Initial denaturation at $95^{\circ} \mathrm{C}$ for $30 \mathrm{sec} ; 40$ cycles of $95^{\circ} \mathrm{C}$ for $5 \mathrm{sec}, 60^{\circ} \mathrm{C}$ for $30 \mathrm{sec}$ and one cycle of melting curve at $95^{\circ} \mathrm{C}$ for $15 \mathrm{sec}, 60^{\circ} \mathrm{C}$ for $1 \mathrm{~min}, 95^{\circ} \mathrm{C}$ for $15 \mathrm{sec}$ and $50^{\circ} \mathrm{C}$ for $30 \mathrm{sec}$. Quantification of METTL3 mRNA expression levels was performed using the $2^{-\Delta \Delta \mathrm{Ct}}$ method (10) with $\beta$-actin as an internal control. The sequences of primers were as follows: METTL3 forward, 5'-ACCCTGACAGATGATGAGATGC-3' and reverse, 5'-CGTTCATACCCCCAGAGGTTTAG-3'; $\beta$-actin forward, 5'-TCCTCCCTGGAGAAGAGCTAC-3' and reverse, 5'-TCCTGCTTGCTGATCCACAT-3.

CCK-8 assay. Cells were seeded into a 96-well plate $\left(3,000\right.$ cells/well) and cultured in DMEM at $37^{\circ} \mathrm{C}$ for 0,24 , 48 or $72 \mathrm{~h}$, and $10 \mu \mathrm{l}$ CCK-8 solution (Beyotime Institute of Biotechnology) was added into each well according to the manufacturer's protocols. After incubation for $2 \mathrm{~h}$ at $37^{\circ} \mathrm{C}$ and $5 \% \mathrm{CO}_{2}$, the absorbance of cells at $450 \mathrm{~nm}$ was detected using a microplate reader.

Colony formation assay. SKOV3 and OVCAR3 cells were seeded in a $6-\mathrm{cm}$ petri dish at the density of 500 cells/well and normally cultured in DMEM at $37^{\circ} \mathrm{C}$ for 14 days. Subsequently, the colonies were fixed with $4 \%$ methanol and stained with $0.1 \%$ crystal violet at $37^{\circ} \mathrm{C}$ for $30 \mathrm{~min}$ (Sigma-Aldrich; Merck $\mathrm{KGaA}$ ). Images of visible colonies were captured with a HP Scanjet G4010 scanner and counted manually.

Flow cytometry detection of cell apoptosis. Transfected SKOV3 and OVCAR3 cells were stained using an Annexin V-FITC Apoptosis Detection kit I (BD Biosciences) according to the manufacturer's protocols. Cells were analyzed using the BD FACS Canto II system (BD Biosciences) and analyzed using FlowJo software version 4.5 (Tree Star, Inc.). Viable cells were negative for both PI and Annexin V, while apoptotic cells were positive for Annexin $\mathrm{V}$ and negative for PI. Late apoptotic dead cells showed both Annexin V and PI positivity.

Transwell invasion assay. Cell invasion was evaluated using Matrigel-coated Transwell chambers at $37^{\circ} \mathrm{C}$ for $24 \mathrm{~h}$ (BD Biosciences). A total of $1 \times 10^{5}$ ovarian cancer cells in $200 \mu \mathrm{l}$ serum-free DMEM were added into the upper chamber. A total of $500 \mu \mathrm{l}$ DMEM with $10 \%$ FBS was added to the lower chamber, and the cells were incubated for $24 \mathrm{~h}$. The non-invasive cells remaining in the upper chamber of the Transwell plate were scraped off with a cotton swab. Invaded cells on the lower surface of the chamber were stained with $0.1 \%$ crystal violet at $25^{\circ} \mathrm{C}$ for $10 \mathrm{~min}$. The cell number was counted as the average of five random fields under a light microscope (Nikon TE2000; Nikon Corporation).

Western blotting. Following transfection for $48 \mathrm{~h}$, cell lysates were prepared using RIPA lysis buffer and protease cocktail inhibitor I (Merck KGaA). The protein was separated using $10 \%$ SDS-PAGE and subsequently transferred to 
Table I. METTL3 expression levels associated with the clinicopathological parameters in ovarian cancer tissues.

METTL3 expression level

\begin{tabular}{|c|c|c|c|c|}
\hline \multirow{2}{*}{ Clinicopathological parameters } & \multirow[b]{2}{*}{$\mathrm{n}$} & & \multirow[b]{2}{*}{ P-value } \\
\hline & & High, n (\%) & Low, n (\%) & \\
\hline \multicolumn{5}{|l|}{ Age, years } \\
\hline$<50$ & 38 & $28(73.7)$ & $10(26.3)$ & 0.863 \\
\hline$\geq 50$ & 14 & $10(71.4)$ & $4(28.6)$ & \\
\hline \multicolumn{5}{|l|}{ Tumor diameter, $\mathrm{cm}$} \\
\hline$<3$ & 23 & $13(56.5)$ & $10(43.5)$ & $0.019^{\mathrm{a}}$ \\
\hline$\geq 3$ & 29 & $25(86.2)$ & $4(13.8)$ & \\
\hline \multicolumn{5}{|l|}{ Lymph node metastasis } \\
\hline Yes & 34 & $29(85.3)$ & $5(14.7)$ & $0.016^{\mathrm{a}}$ \\
\hline No & 18 & $9(50)$ & $9(50)$ & \\
\hline \multicolumn{5}{|l|}{ Pathological grading } \\
\hline I-II & 13 & $6(12.9)$ & $7(87.1)$ & $0.030^{\mathrm{a}}$ \\
\hline III-IV & 39 & $32(56.5)$ & $7(43.5)$ & \\
\hline \multicolumn{5}{|l|}{ Histotype } \\
\hline Serous adenocarcinoma & 17 & $14(82.4)$ & $3(17.6)$ & 0.613 \\
\hline Mucinous adenocarcinoma & 16 & $11(68.8)$ & $5(31.2)$ & \\
\hline Endometrioid adenocarcinoma & 12 & $8(66.7)$ & $4(33.3)$ & \\
\hline Clear cell carcinoma & 7 & $5(71.43)$ & $2(28.57)$ & \\
\hline
\end{tabular}

${ }^{a} \mathrm{P}<0.05$. The pathological grade was staged according to the International Union against Cancer/American Joint Committee on Cancer system (11). METTL3, methyltransferase-like 3.

a PVDF membrane. After blocking in a 5\% non-fat milk for $1 \mathrm{~h}$, the membrane was washed with TBS $+0.1 \%$ Tween-20. Membranes were incubated with primary antibodies against METTL3 (1:1,000; cat. no. GTX105037; GeneTex, Inc.), Bcl-2 (1:2,000; cat. no. 60178-1-Ig; ProteinTech Group, Inc.); Bax (1:1,000; cat. no. 50599-2-Ig; ProteinTech Group, Inc.); active caspase 3 (1:1,000; rabbit polyclonal antibody; cat. no. 19677-1-AP; ProteinTech Group, Inc.); p-AKT (1:1,000; cat. no. 66444-1-Ig; ProteinTech Group, Inc.); AKT (1:500; cat. no. 9272; Cell Signaling Technology, Inc.); p70S6K (1:1,000; cat. no. GTX107562; GeneTex, Inc.); Cyclin D1 (1:1,000; cat. no. GTX108624; GeneTex, Inc.) and tubulin (1:1,000; cat. no. GTX76511; GeneTex, Inc.) at $4^{\circ} \mathrm{C}$ overnight, followed by incubation with anti-rabbit $\operatorname{IgG}(1: 2,000$; cat. no. GTX300119; GeneTex, Inc.) or anti-mouse IgG (1:2,000; cat. no. GTX300120; GeneTex, Inc.) secondary antibodies for $1 \mathrm{~h}$ at room temperature. Protein bands were visualized using Pierce ECL Western Blotting Substrate (Thermo Fisher Scientific, Inc.). Protein band intensity was analyzed using Image J software, v1.41 (National Institutes of Health).

Statistical analysis. Data analysis was performed using GraphPad Prism 7 (GraphPad Software, Inc.), and all experiments were performed in triplicate. Differences between two groups were evaluated using Student's t-test; one-way ANOVA was used to analyze multiple groups, followed by Tukey's post-hoc test. $\mathrm{P}<0.05$ was considered to indicate a statistically significant difference.

\section{Results}

METTL3 expression levels are associated with clinical parameters in patients with ovarian cancer. METTL3 expression levels were significantly highly in serous adenocarcinoma, mucinous adenocarcinoma, endometrioid adenocarcinoma and clear cell carcinoma tissues compared with corresponding paracancerous tissues. METTL3 expression levels were not associated with different histotypes of ovarian cancer tissues. High METTL3 expression levels were associated with large tumor size $(\mathrm{P}=0.0188)$, lymph node metastasis $(\mathrm{P}=0.0163)$ and high pathological grade $(\mathrm{P}=0.0303)$. The pathological grade was staged according to the International Union against Cancer/American Joint Committee on Cancer system (11). These data indicated that METTL3 expression levels may be associated with the tumor growth and metastasis of ovarian cancer (Fig. 1 and Table II).

Downregulation of METTL3 inhibits proliferation and colony formation in human ovarian cancer tissues. To investigate the biological function of METTL3 in human ovarian cancer, loss-of-function assays were performed. The interference efficiency results demonstrated that shRNA1, shRNA2 and shRNA3 targeting METTL3 significantly downregulated METTL3 mRNA expression levels in SKOV3 and OVCAR3 cells compared with negative control group $(\mathrm{P}<0.05$; Fig. 2A and B). shRNA3 in SKOV3 cells and shRNA1 in OVCAR3 cells (shMETTL3) were selected for further experiments due to the interference efficiencies, and knockdown 
Table II. METTL3 expression levels in ovarian cancer and para-carcinoma tissue.

METTL3 expression level

\begin{tabular}{lcccr} 
Tissue & $\mathrm{n}$ & Low, n (\%) & High, n (\%) & P-value \\
\hline Ovarian cancer & 52 & $14(18.3)$ & $38(81.7)$ & $<0.001^{\mathrm{a}}$ \\
Para-carcinoma & 52 & $40(87.1)$ & $12(12.9)$ & $<0.001^{\mathrm{a}}$ \\
\hline
\end{tabular}

${ }^{\text {aP }}<0.05$. METTL3, methyltransferase-like 3.

A

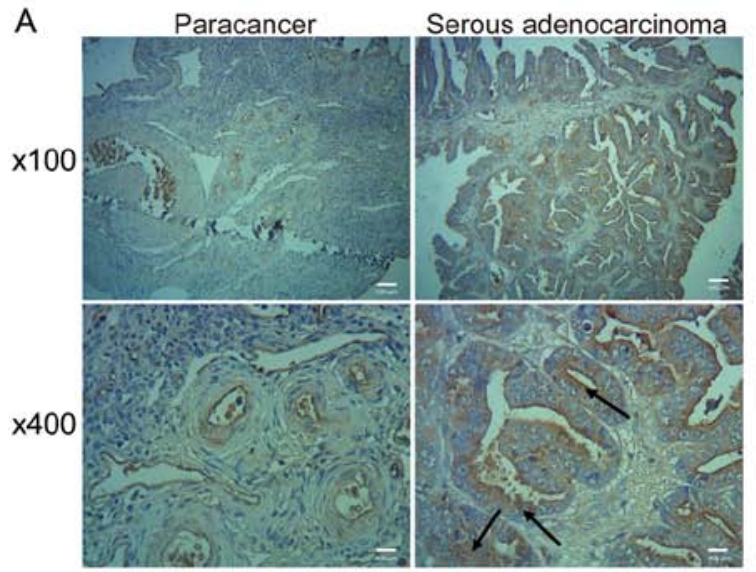

C

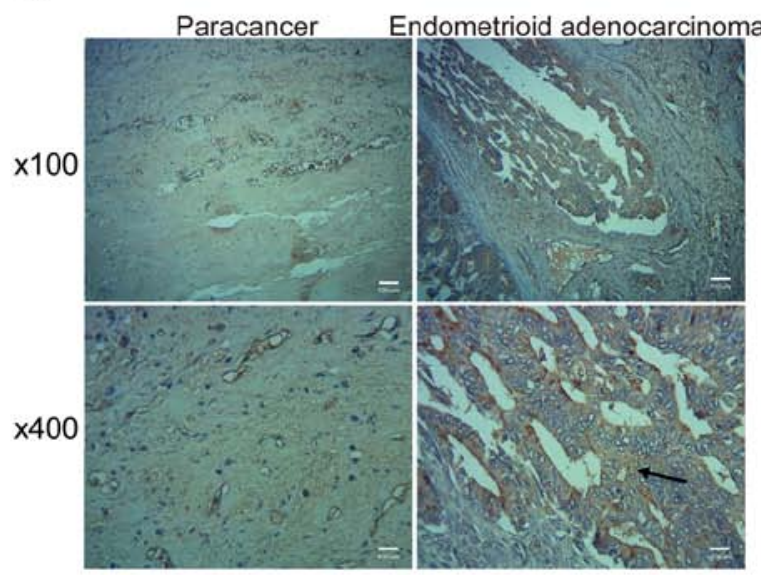

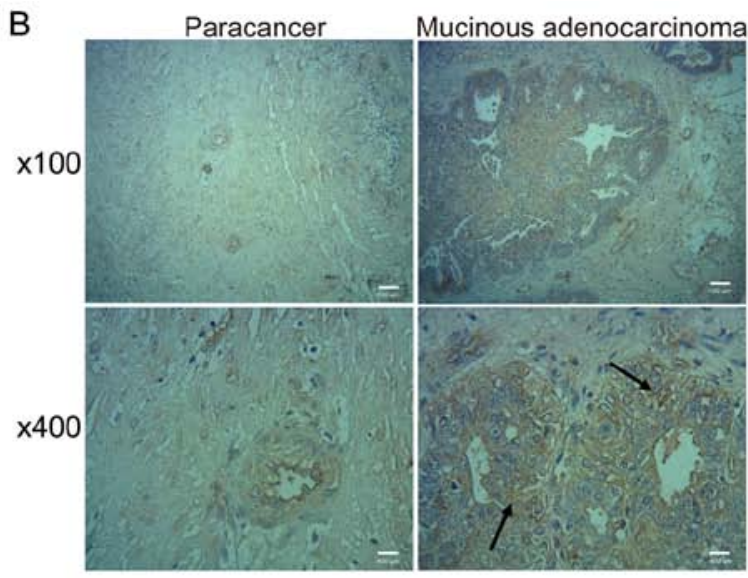

D

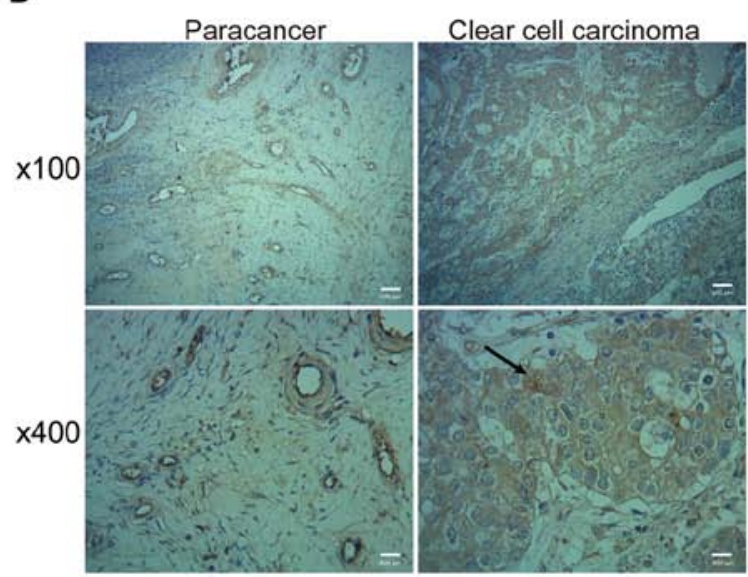

Figure 1. METTL3 expression levels are high ovarian cancer tissues. Areas indicated by arrows indicate sites of high METTL3 expression levels. (A-D) Immunohistochemical staining of METTL3 in ovarian cancer tissues and para-carcinoma in cases of: (A) serous adenocarcinoma, (B) mucinous adenocarcinoma, (C) endometrioid adenocarcinoma and (D) clear cell carcinoma. Magnifications, x100 and x400. Scale bars, 100 and $400 \mu \mathrm{m}$. METTL3, methyltransferase-like 3 .

efficacy was validated at the protein level (Fig. 2C and D). The cell proliferation assay results indicated that METTL3 knockdown significantly inhibited the proliferation of SKOV3 and OVCAR3 cells (Fig. 3A and $\mathrm{B} ; \mathrm{P}<0.05$ ). In addition, cell colony number was also significantly decreased when METTL3 expression was silenced (Fig. 3C and D).

METTL3 knockdown induces apoptosis and may lead to the activation of the mitochondrial apoptosis pathway in ovarian cancer cells. Apoptosis analysis results suggested that the percentage of SKOV3 and OVCAR3 cells undergoing apoptosis was significantly increased when METTL3 expres- sion levels were knocked down compared with the control at $48 \mathrm{~h}$ and $72 \mathrm{~h}$ (Fig. 4A-D). METTL3 knockdown also led to a significant upregulation of the pro-apoptotic protein Bax and downstream effector Caspase 3 expression levels, whereas the expression of the anti-apoptotic protein $\mathrm{Bcl} 2$ was downregulated (Fig. 4E and F). These data suggested that METTL3 knockdown activated the mitochondrial apoptosis pathway in SKOC3 and OVCAR3 cells.

METTL3 knockdown inhibits cell invasion and may reduce the activation of the AKT signaling pathway. The effect of METTL3 knockdown on the invasive ability of SKOV3 and 

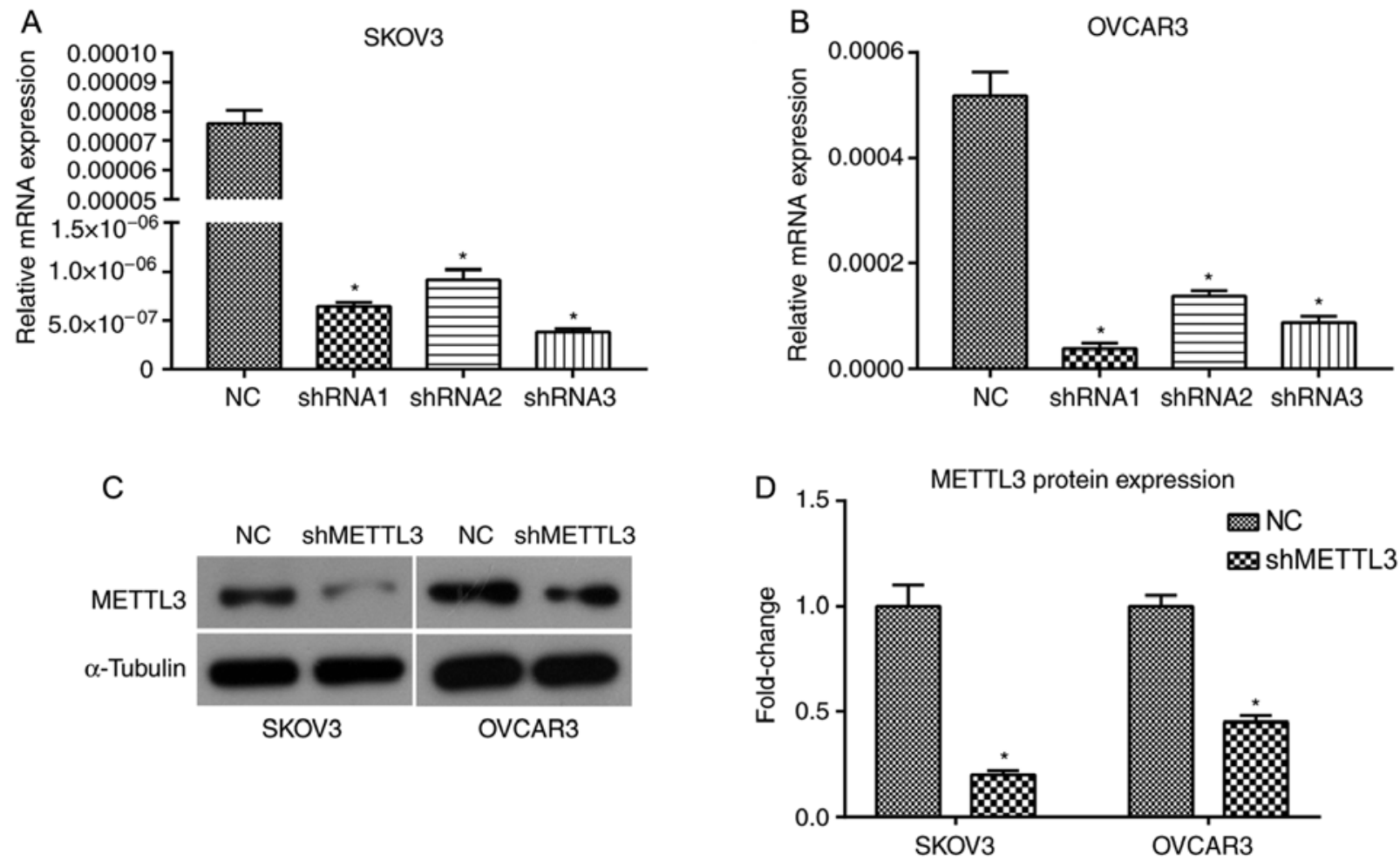

Figure 2. METTL3 knockdown in SKOV3 and OVCAR3 ovarian cancer cells mediated by shRNA transfection. All experiments were performed in triplicate. shRNA1, shRNA2, shRNA3, shMETTL3 or a negative control shRNA was introduced into SKOV3 and OVCAR3 cells. METLL3 mRNA expression levels in (A) SKOV3 and (B) OVCAR3 cells treated with shRNA1, shRNA2, shRNA3 or NC. (C) METTL3 protein expression levels in SKOV3 and OCVAR3 cells treated with shRNA3 and shRNA1, respectively. (D) Quantified METTL3 protein expression levels using ImageJ software in SKOV3 and OVCAR3 cells. "P<0.05 vs. NC. METTL3, methyltransferase-like 3; sh, short hairpin; NC, negative control; shMETTL3, shRNA3 in SHOV3 cells or shRNA1 in OVCAR3 cells.

A

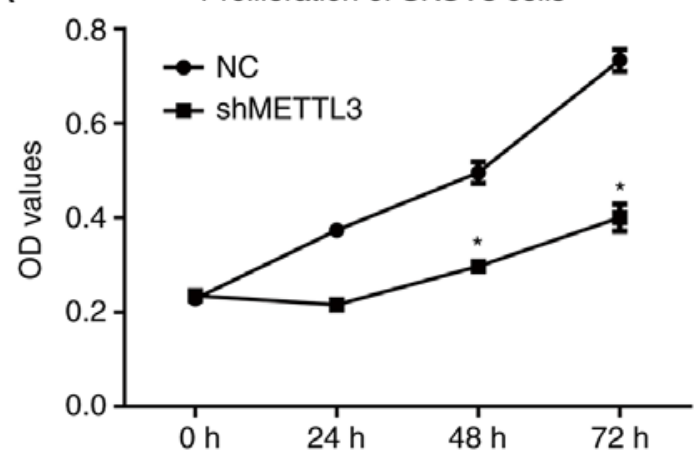

B

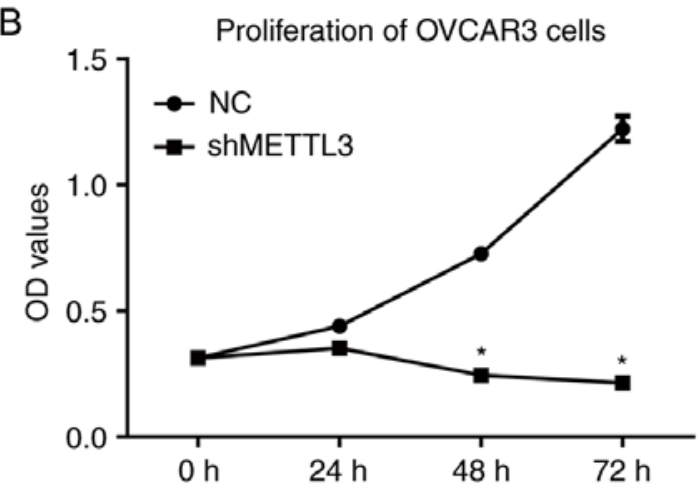

C

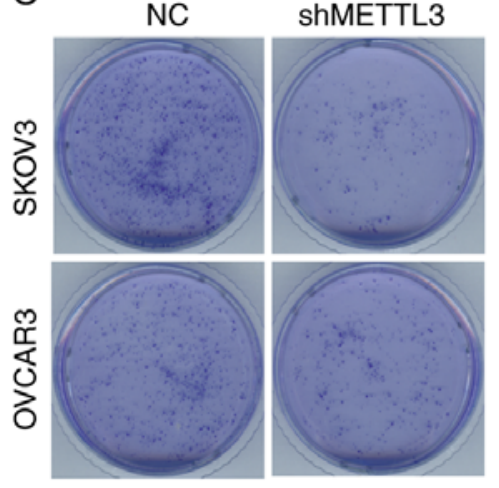

D

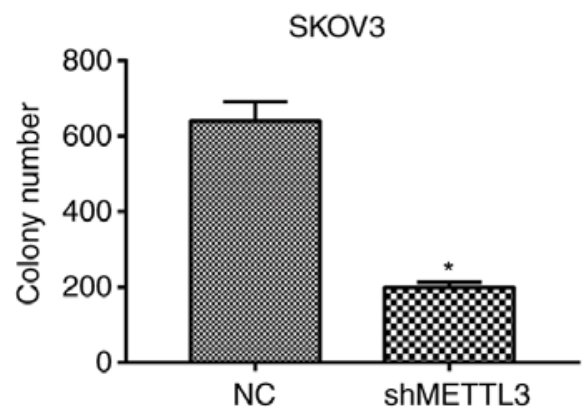

Figure 3. Downregulation of METTL3 expression inhibits proliferation and colony formation of human ovarian cancer cells. (A) Proliferation of SKOV3 cells treated with shMETTL3 or NC at different time points. (B) Proliferation of OVCAR3 cells treated with shMETTL3 or NC at different time points. (C and D) Colony formation of SKOV3 and OVCAR3 cells treated with shMETTL3 or NC. All experiments were performed in triplicate. ${ }^{*} \mathrm{P}<0.05$ vs. NC. METTL3, methyltransferase-like 3; sh, short hairpin; NC, negative control. 
A
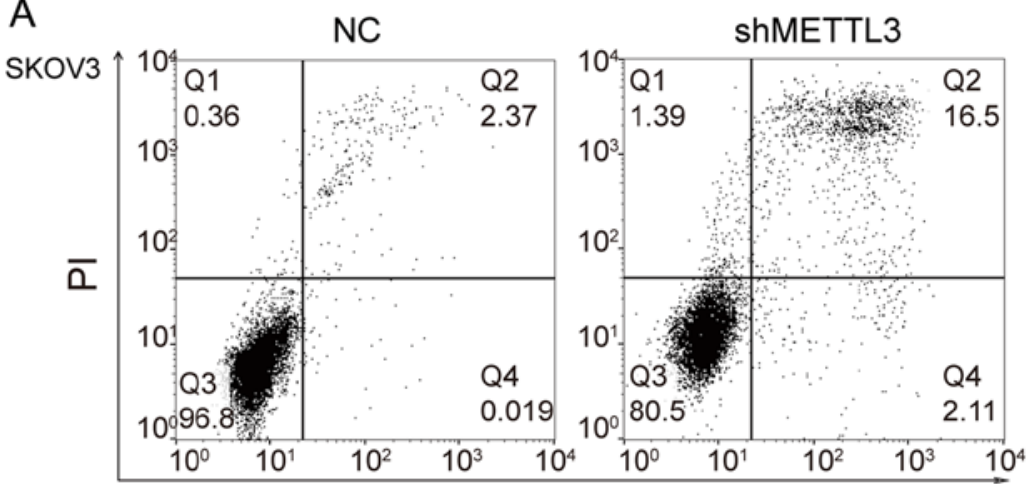

Annexin $\mathrm{V}$

C

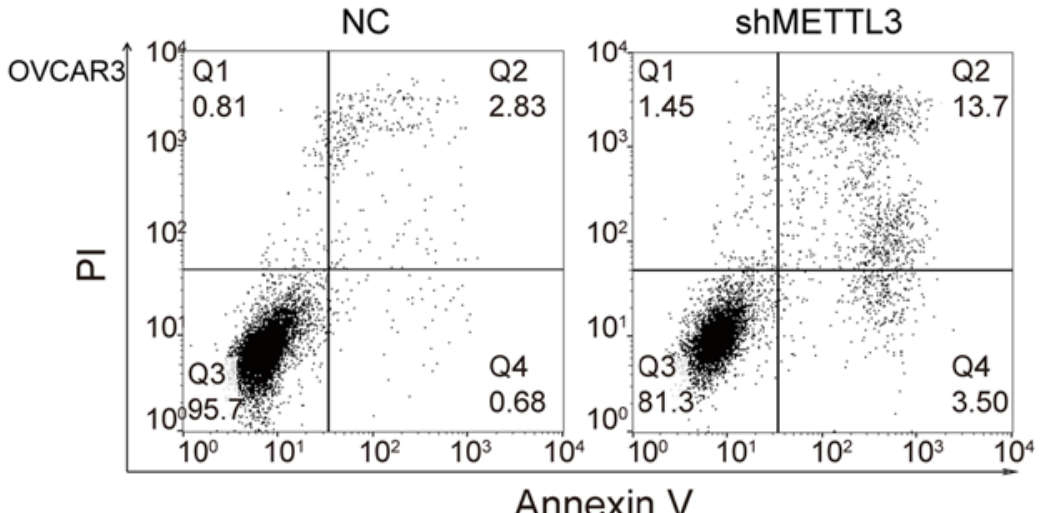

B

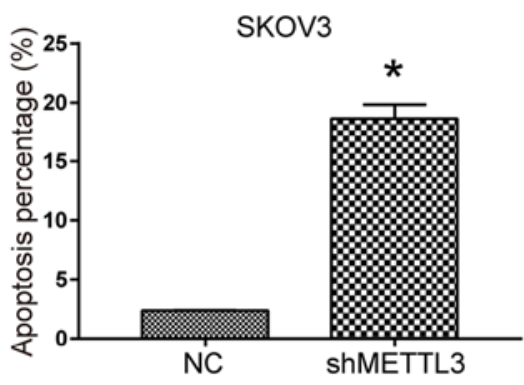

D

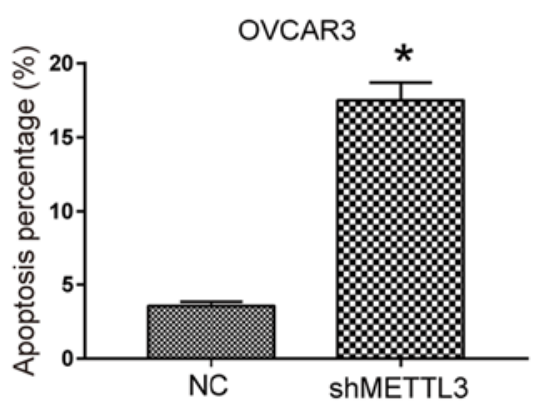

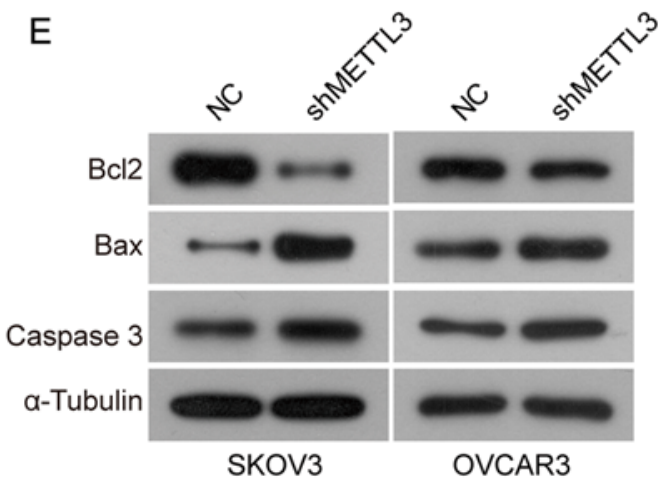

$\mathrm{F}$
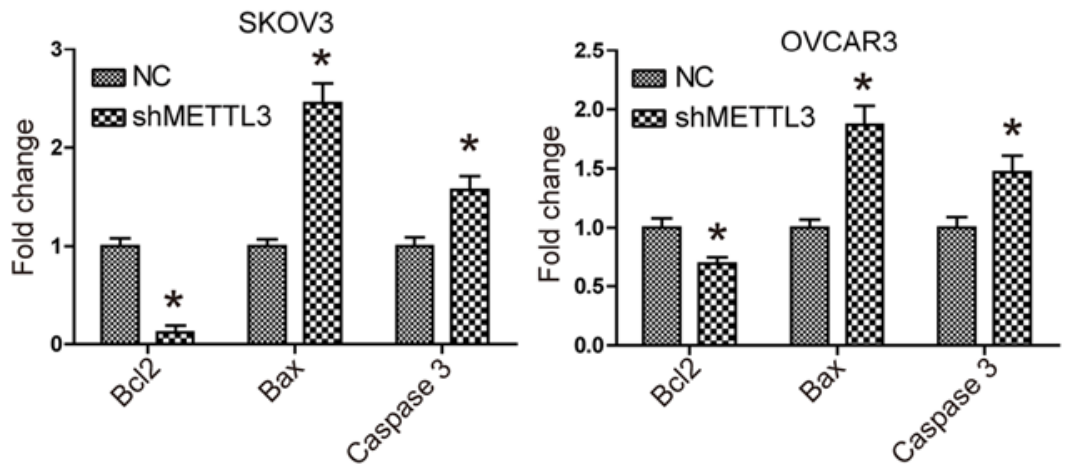

Figure 4. Downregulation of METTL3 expression increases apoptosis in human ovarian cancer cells. (A and B) Quantification of Annexin V/PI staining in SKOV3 cells transfected with shMETTL3 or NC. (C and D) Quantification of Annexin V/PI staining in OVCAR3 cells transfected with shMETTL3 or NC. The data were analyzed by using FlowJo version 10 software. (E and F) Bcl2, Bax and Caspase 3 protein expression levels in SKOV3 and OVCAR3 cells with treated with shMETTL3 or NC. All experiments were performed in triplicate. ${ }^{*} \mathrm{P}<0.05$ vs. NC. METTL3, methyltransferase-like 3; sh, short hairpin; NC, negative control; PI, propidium iodide.

OVCAR3 cells was investigated using Transwell assays. The invasive ability of SKOV3 and OVCAR3 cells was significantly reduced when METTL3 expression levels were knocked down compared with the control $(\mathrm{P}<0.05$; Fig. 5A and B). It was hypothesized that thr AKT signaling pathway may be associated with the biological function of METTL3. METTL3 knockdown led to decreased expression levels of phosphorylated AKT and its downstream effectors p70S6K and Cyclin D1 (Fig. 5C and D). These results suggested that knockdown of METTL3 expression led to reduced activation of the AKT signaling pathway in ovarian cancer cells.

\section{Discussion}

In the present study, METTL3 expression levels in ovarian cancer tissues were investigated; the results demonstrated that knockdown of METTL3 significantly inhibited proliferation, colony formation and invasion of ovarian cancer cells. In addition, the apoptotic rate was increased when METTL3 expression levels were knocked down. Downregulation of METTL3 increased the expression levels of the pro-apoptotic Bax and Caspase 3, whereas the expression levels of the anti-apoptotic Bcl2 were decreased. During apoptosis, Bax binds to the mitochondrial outer 
A

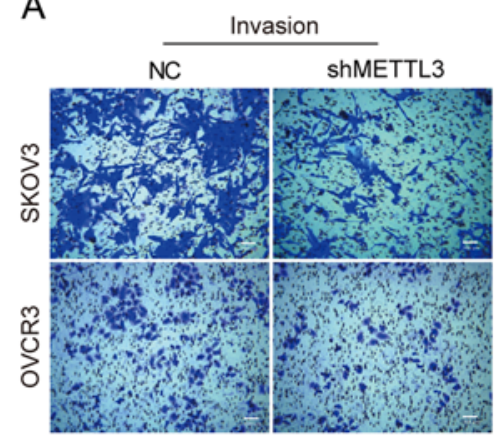

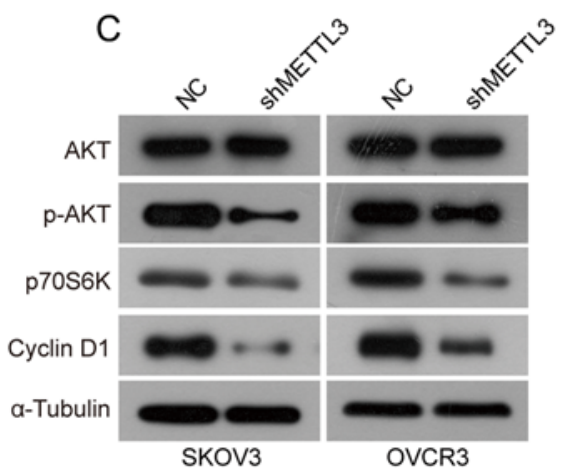

B

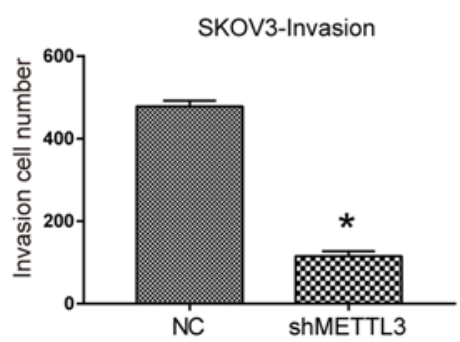

D
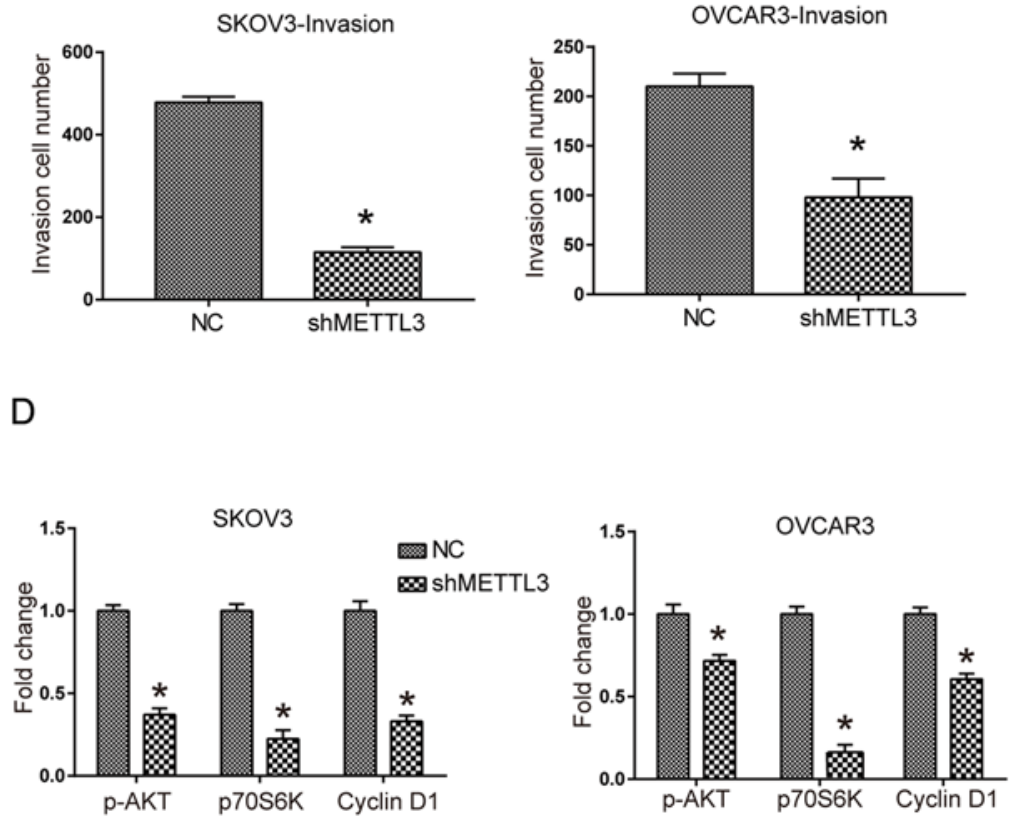

NC $\otimes$ ShMETTL3

Figure 5. METTL3 knockdown inhibits cell invasion and may reduce the activation of the AKT signaling pathway. (A and B) Quantified cell migration and invasion of SKOV3 and OVCAR3 cells treated with shMETTL3 or NC. (C and D) Expression of the components of the AKT signaling pathway, including AKT, p-AKT, p7026K and Cyclin D1, in SKOV3 and OVCR3 cells treated with shMETTL3 or NC. All experiments were performed in triplicate. Scale bar, $100 \mu \mathrm{m} .{ }^{*} \mathrm{P}<0.05$ vs. NC. METTL3, methyltransferase-like 3; sh, short hairpin; NC, negative control; p-, phosphorylated.

membrane and promotes its permeability, causing the release of cytochrome $\mathrm{c}$ into the cytoplasm, which induces the activation of Caspase 3 (12). By contrast, Bcl2 functions as an apoptosis inhibitor and blocks the promotion of mitochondrial permeability (13). To the best of our knowledge, the present study demonstrated the oncogenic function of METTL3 in the biological process of ovarian cancer cells for the first time.

An increasing number of studies have demonstrated the function of METTL3 in tumor formation and progression. For example, Lin et al (7) have reported that METTL3 promotes the translation of epidermal growth factor receptor and the Hippo pathway effector TAZ in human lung cancer cells. In addition, reduced METTL3 expression levels inhibit tumor growth and metastasis to the lung of hepatocellular carcinoma (HCC) in vitro and in vivo (14). Du et al (15) have demonstrated that microRNA-33a functions as a tumor suppressor in non-small-cell lung carcinoma cells, suppressing the translation of METTL3 mRNA. However, another study identified METTL3 as a tumor suppressor in renal cell carcinoma, inhibiting tumor proliferation, migration and cell cycle progression (9). These results from previous studies suggest that the functions of METTL3 can vary between different tumor types, and a possible explanation for this may be the high degree of tumor heterogeneity or gene mutation-induced function alteration.

The modes of METTL3 action can be divided into two types: m6A-dependent and m6A-independent $(14,16)$. For example, METTL3 depletion can promote suppressor of cytokine signaling 2 expression by decreasing m6A methyl- ation-mediated degradation, thus blocking the progression of HCC (14). Cai et al (16) demonstrated that METTL3-induced m6A modification increased the expression levels of hepatitis $\mathrm{B}$ X-interacting protein, thus promoting the proliferation of breast cancer cells. METTL3 has also been identified to elevate oncoprotein expression levels in tumor cells by functioning as a transcription enhancer factor or promoting the assembly of mRNA translation machinery (7). In the present study, the mechanisms underlying the oncogenic function of METTL3 in human ovarian cancer were investigated. A previous study reported that the AKT signaling pathway regulates numerous biological processes, such as promoting cell proliferation and survival (17). The present study demonstrated that METTL3 downregulation decreased the expression levels of phosphorylated AKT and its downstream effectors, including p70S6K and Cyclin D1, indicating reduced activation of the AKT pathway. However, the present study was unable to identify the direct target of METTL3 function in human ovarian cancer cell lines due to the experimental conditions.

In conclusion, METTL3 knockdown inhibits tumorigenesis and tumor progression of human ovarian cancer cells in vitro, which may be mediated by reduced activation of the AKT signaling pathway. These results may provide novel insight into the potential targeting of METTL3 in ovarian cancer treatment.

\section{Acknowledgements}

Not applicable. 


\section{Funding}

The present study was supported by The National Natural Science Foundation of China (grant no. 81503298) and Natural Science Foundation of Shandong Province (grant no. ZR2014HM008).

\section{Availability of data and materials}

The datasets used and/or analyzed during the present study are available from the corresponding author upon reasonable request.

\section{Authors' contributions}

SL designed the study. SL, HG, XL, NL, FG and JL performed experiments and analyzed data. SL wrote the manuscript. All authors read and approved the final manuscript.

\section{Ethical approval and consent to participate}

The present study was approved by The Ethics Committee of Shandong Provincial Hospital (China). All patients provided written informed consent.

\section{Patient consent for publication}

Not applicable.

\section{Competing interests}

The authors declare that they have no competing interests.

\section{References}

1. Torre LA, Trabert B, DeSantis CE, Miller KD, Samimi G, Runowicz CD, Gaudet MM, Jemal A, and Siegel RL: Ovarian cancer statistics, 2018. CA Cancer J Clin 68: 284-296, 2018.

2. Scholz HS, Tasdemir H, Hunlich T, Turnwald W, Both A and Egger H: Multivisceral cytoreductive surgery in FIGO stages IIIC and IV epithelial ovarian cancer: Results and 5-year follow-up. Gynecol Oncol 106: 591-595, 2007.

3. Wei W, Ji X, Guo X and Ji S: Regulatory role of N6 -methyladenosine (m6 A) methylation in RNA processing and human diseases. J Cell Biochem 118: 2534-2543, 2017.
4. Wang X, Lu Z, Gomez A, Hon GC, Yue Y, Han D, Fu Y, Parisien M, Dai Q, Jia G, et al: N-6-methyladenosine-dependent regulation of messenger RNA stability. Nature 505: 117-120, 2014.

5. Lin S and Gregory RI: Methyltransferases modulate RNA stability in embryonic stem cells. Nat Cell Biol 16: 129-131, 2014.

6. Schwartz S, Mumbach MR, Jovanovic M, Wang T, Maciag K, Bushkin GG, Mertins P, Ter-Ovanesyan D, Habib N, Cacchiarelli D, et al: Perturbation of m6A writers reveals two distinct classes of mRNA methylation at internal and 5 ' sites. Cell Rep 8: 284-296, 2014.

7. Lin S, Choe J, Du P, Triboulet R and Gregory RI: The m(6)A methyltransferase METTL3 promotes translation in human cancer cells. Mol Cell 62: 335-345, 2016.

8. Vu LP, Pickering BF, Cheng Y, et al: The N-6-methyladenosine (m(6)A)-forming enzyme METTL3 controls myeloid differentiation of normal hematopoietic and leukemia cells. Nat Med 23: 1369-1376, 2017.

9. Li X, Tang J, Huang W, Wang F, Li P, Qin C, Qin Z, Zou Q, Wei J, Hua L, et al: The M6A methyltransferase METTL3: Acting as a tumor suppressor in renal cell carcinoma. Oncotarget 8: 96103-96116, 2017.

10. Livak KJ and Schmittgen TD: Analysis of relative gene expression data using real-time quantitative PCR and the 2(- $\Delta \Delta \mathrm{C}(\mathrm{T}))$ Method. Methods 25: 402-408, 2001.

11. Kroeger PT Jr and Drapkin R: Pathogenesis and heterogeneity of ovarian cancer. Curr Opin Obstet Gynecol 29: 26-34, 2017.

12. Yi X, Yin XM and Dong Z: Inhibition of Bid-induced apoptosis by $\mathrm{Bcl}-2$. tBid insertion, Bax translocation, and Bax/Bak oligomerization suppressed. J Biol Chem 278: 16992-16999, 2003.

13. Radha $G$ and Raghavan SC: BCL2: A promising cancer therapeutic target. Biochimica et Biophysica Acta (BBA) -. Rev Can 1868: 309-314, 2017.

14. Chen M, Wei L, Law C-T, et al: RNA N6-methyladenosine methyltransferase-like 3 promotes liver cancer progression through YTHDF2-dependent posttranscriptional silencing of SOCS2. Hepatology 67: 2254-2270, 2017.

15. Du M, Zhang Y, Mao Y, Mou J, Zhao J, Xue Q, Wang D, Huang J, Gao S and Gao Y: MiR-33a suppresses proliferation of NSCLC cells via targeting METTL3 mRNA. Biochem Biophys Res Commun 482: 582-589, 2017.

16. Cai X, Wang X, Cao C, Gao Y, Zhang S, Yang Z, Liu Y, Zhang $X$, Zhang $W$ and Ye L: HBXIP-elevated methyltransferase METTL3 promotes the progression of breast cancer via inhibiting tumor suppressor let-7g. Cancer Lett 415: 11-19, 2018.

17. Martini M, De Santis MC, Braccini L, Gulluni F and Hirsch E: PI3K/AKT signaling pathway and cancer: An updated review. Ann Med 46: 372-383, 2014. 